Relations industrielles

Industrial Relations

\title{
A Behavioral Theory of the Firm, par Richard M. Cyert et James G. March, Prentice-Hall, Inc., Englewood Cliffs, New Jersey, 1963, 332 pages.
}

\section{Bertrand Belzile}

Volume 19, numéro 3, juillet 1964

URI : https://id.erudit.org/iderudit/1021288ar

DOI : https://doi.org/10.7202/1021288ar

Aller au sommaire du numéro

Éditeur(s)

Département des relations industrielles de l’Université Laval

ISSN

0034-379X (imprimé)

1703-8138 (numérique)

Découvrir la revue

Citer ce compte rendu

Belzile, B. (1964). Compte rendu de [A Behavioral Theory of the Firm, par Richard M. Cyert et James G. March, Prentice-Hall, Inc., Englewood Cliffs, New Jersey, 1963, 332 pages.] Relations industrielles / Industrial Relations, 19(3), 409-410. https://doi.org/10.7202/1021288ar

Tous droits réservés (C Département des relations industrielles de l’Université Laval, 1964
Ce document est protégé par la loi sur le droit d'auteur. L'utilisation des services d’Érudit (y compris la reproduction) est assujettie à sa politique d'utilisation que vous pouvez consulter en ligne.

https://apropos.erudit.org/fr/usagers/politique-dutilisation/ 
Bénélux, Union de l'Europe occidentale, Conseil de I'Europe, Organisation européenne de coopération économique, O.C.D.E., Marchés communs, ainsi qu'une foule d'autres institutions témoignent de cette cohésion europétnne naissante.

Mais le nombre, la diversité et la fragmentation sur le plan des moyens et des objectifs de ces instruments laissent voir oussi à quel point leur développement s'est foit à partir d'un empirisme qui laisse peu de place ou rationnel et à la logique des systèmes juridiques traditionnels. Nous sommes réellement en face d'un phénomène avant tout d'ordre sociologique, et seule une sociologie du droit, encore en gestation, peut rendre compte volablement d'un tel phénomène.

L'outeur n'o pas eu lo prétention de faire un traité de sociologie du droit ni même de présenter un traité définitif de droit social européen, puisqu'il prend la peine de bien spécifier sur la poge couverture et dans son introduction qu'il ne s'ogit tout ou plus que d'une «édition préliminaire $\gg$, loissant ou bouillonnement octuel le temps de roder les institutions et leur fonctionnement avont de songer à en tirer une analyse théorique et un système formel plus complets

Les Eléments de droit social européen constitue, en fait un premier stage vers l'élaboration d'une sociologie du droit véritable; il est un effort de collection et de présentation ordonnée des textes et des instruments existont présentement en droit social à l'échelle de l'Europe ou à tout le moins de certains pays de ce continent. $\mathrm{Ce}$ seul mérite justifie les plus grands éloges.

L'ouvroge comprend trois parties: o) les cadres institutionnels, où sont présentés, dons leurs origines, leur développement historique et leur organisotion, les principoles intstitutions étudiées; b) les instruments juridiques, où l'auteur fait la présentation et étudie les coractères et la nature des accords, conventions, résolutions, règlements etc. . qui servent d̀ mettre en oeuvre les institutions déjà décrites; c) les problèmes socioux et les solutions, qui est la partie essentielle de l'ouvrage, et où l'auteur a regroupé l'étude des institutions selon les ordres de problèmes qu'ils tendent à résoudre, afin d'en dégoger les lignes de force d'un véritable droit social européen par la comparaison des solutions apportées par les divers instruments à un même problème: les droits sociaux fondamentoux, les réfugiés et apatrides, la libre circulation des travailleurs et l'emploi, la rémunérotion, l'ossis- tance sociale et médicale, la sécurité sociole, la formation professionnelle, sont étudiés ò tour de rôle. Ainsi se dégagent les éléments d'un effort convergent entre les différentes institutions européennes tendant c̀ créer finclement un droit social cohérent ò l'échelle de ce continent.

Un traité à lire et à consulter pour tout observateur de la chose européenne à l'heure présente

\section{Jean-Réal Cardin}

A Behovioral Theory of the Firm, por Richard M. Cyert et James G. March, Prentice-Hall, Inc., Englewood Cliffs, New Jersey, 1963, 332 pages.

Les décisions constituent certainement l'activité névralgique des odministrateurs des organisations et particulièrement des entreprises. C'est ainsi que la < science administrative s'oriente de plus en plus vers l'étude du processus de prise de décision. Le présent ouvroge démontre précisément l'opport précieux du «behoviorisme », ou science du comportement, dans l'établissement d'une théorie de la décision. Les auteurs croient que pour comprendre la prise de décision économique contemporaine, il est nécessaire d'ajouter à l'étude des facteurs de marché un examen du fonctionnement interne de l'entreprise, étudier les effets de la structure de l'orgo. nisation et de la pratique conventionnelle sur le développement des buts, la formation des expectatives et l'exécution des choix.

Lo recherche qui o conduit ò ce livre se caractérise de quatre façons: 1) accent sur un petit nombre de décisions économiques clefs prises por l'entreprise; 2) développement de modèles de la firme orientés vers les processus; 3) liaison des modèles oussi près que possible ovec des observo. tions empiriques et 4 ) développement d'une théorie dont la généralité déposse les firmes spécifiques étudiées

Au cours de l'étude, l'emphase fut placée sur les processus de décision actuels. Ceci implique une description de la décision de la firme en termes d'une série spécifique d'étapes franchies pour en orriver à cette décision.

Le codre de référence fondamental d'anolyse que les outeurs proposent, comme le cadre clossique, possède deux devis majeurs d'organisation: d'abord un ensemble de catégories exhoustives de variables et ensuite un ensemble de concepts relationnels. Les outeurs prétendent pouvoir analyser le pro- 
cessus de la prise de décision dans l'entreprise moderne en termes de variables qui offectent les buts, les expectatives et le choix de l'organisation. Les concepts relationnels, qui représentent ò plusieurs aspects le coeur de leur théorie de la décision, sont les suivonts: 1) «quosi resolution of conflict \$2) \&uncertainty ovoidonce 》3) «problemistic search: \$, 4) * organizational learning $\gg$.

A partir de ce cadre d'onalyse, les auteurs bâtissent un nombre de modèles et en suggèrent les implications pour la théorie économique et celle de l'organisation. Selon eux, le langoge théorique naturel dans ce contexte est celui du programme d'un ordinateur. Au chapitre 7 , on trouve un modèle de la déterminatión du prix et de la production dans un magasin à rayons. Au chapitre suivant, les auteurs exposent un modèle général de détermination du prix et de la production dans un oligopole; de plus, ils examinent quelques propriétés du modèle. Dans les chapitres 9 et 10, on peut prendre connaissance de deux modèles: le premier, du comportement administratif rationel et l'outre, du comportement d'une société de fiducie dans l'investissement.

Tout en n'étont pas très facile, ce volume ouvre des horizons nouveaux sur le comportement des administrateurs, particuiièrement dons le domaine du processus de la prise de décision, et partant présente un intérêt certain pour la plupart de ceux qui s'intéressent à l'administration de l'entreprise.

\section{Bertrand Belzile}

\section{Economic Aspects of Education, par W.C. Bowen, Industrial Relotions Section, Princeton University, 1964.}

Dons une province où les problèmes posés por un changement considérable dans la demande de services d'éducation ont causé et causent encore des perturbotions appréciables, les trois excellents essais du professeur W.C. Bowen devraient intéresser un bon nombre de personnes.

Le premier de ces essais est une évoluotion des différentes méthodes utilisées par les économistes pour mesurer le rendement économique des investissements dons le secteur de l'éducation. II devrait intéresser non seulement les économistes, mais tous ceux qui ont soit à élaborer les politiques d'éducation, soit à les accepter ou les refuser. Soulignons plus particulièrement lo section qui troite des projections de maind'oeuvre comme guide pour les planificoteurs.
Le deuxième essoi traite du financement des universités en Gronde-Bretagne et en France. L'analyse qu'on y fait est cependant d'application universelle puisqu'elle réfère à des problèmes qui sont pour nous d'un intérêt aussi actuel que l'efficacité du système universitaire, l'importance du revenu de la nation qu'on doit y offecter, le recrutement des professeurs, l'équilibre que I'on doit tenir entre les humanités et les sciences, l'enseignement et lo recherche, etc.

Dans le troisième essai, l'auteur discute des disporités de traitements dans les universités anglaises et tente d'en apprécier I'utilité et les conséquences. Ceux qui réalisent que le mécanisme des prix est un puissont facteur d'allocation des ressources humaines autant que physiques en trouveront une excellente illustration ici. Ceux qui ont la responsabilité de recruter les professeurs y trouveront d'utiles informations capables de faciliter l'élaboration de leur politique de recrutement.

\section{Jacques St-Lourent}

\section{Ethics ans Standards in American Business,} par Joseph W. Towle and others. Houghton Mifflin Co., Boston, 1964. 1964. 315 poges.

Nombreux volumes et articles ont été publiés aux Etats-Unis à la suite de l'ouvrage de H.R. Bowen sur la responsabilité sociale des administrateurs et hommes d'offaires. Qu'il suffise de mentionner ici les livres et articles de Berle et Means, Dempsey et Duff. L'intérêt porté aux problèmes de la moralité et la responsabilité sociale en affaires trouve une explication dans les changements suivants: le déclin de la concurrence pure pour foire place ò l'oligopole, la séparation entre la possession des moyens de production et leur contrôle, l'intervention des gouvernements dans l'orientation de l'économie, la tendance ò la professionalisation chez les administrateurs, les tentatives de collusion pour fixer les prix et le renouveau dans l'Eglise en matière économique et sociale.

L'ouvrage que nous recensons porte sur les problèmes moraux qui ont occasionné ces changements. C'est le compte-rendu d'un symposium tenu ò l'Université de Washington, ouquel participaient des représentants des milieux ocodémiques, administrotifs et religieux.

La présentation du motériel tente d'établir une certaine unité entre des exposés qui ne sont pas tous d'égale voleur et qui véhiculent des points de vue antithétiques. 